\title{
THE ENVIRONMENTS OF TYPE IB/C SUPERNOVAE
}

\author{
S. D. VAN DYK, A. J. BARTH AND A. V. FILIPPENKO \\ Astronomy Department, University of California, \\ Berkeley CA 94720-3411 USA
}

\section{Introduction}

Up to about 1985, supernovae (SNe) generally were placed into the two Minkowski classes, type I and type II, defined by the absence or presence, respectively, of hydrogen in their optical spectra. Around that time it was acknowledged that several type I SNe were systematically peculiar, both spectroscopically and photometrically (Elias et al. 1985; Wheeler \& Levreault 1985; Uomoto \& Kirshner 1985; Branch 1986; Filippenko 1986), by missing the characteristic Si II spectral feature near $6150 \AA$, having distinct infrared light curves, being optically redder and subluminous, and showing radio emission (Sramek et al. 1984). These SNe were designated as type $\mathrm{Ib}$ (Elias et al. 1985; Branch 1986) to distinguish them from the classical type Ia. Harkness et al. (1987) identified He I lines in spectra of the $\mathrm{SN} \mathrm{Ib} \mathrm{1984L,} \mathrm{but} \mathrm{some} \mathrm{subsequent} \mathrm{examples} \mathrm{showed} \mathrm{no} \mathrm{He} \mathrm{in} \mathrm{their} \mathrm{spectra}$ and were further subclassified as Type Ic (Wheeler \& Harkness 1990). The two subtypes, however, are nearly indistinguishable at late times. In this Symposium the entire class has been referred to as type $\mathrm{Ib} / \mathrm{c}$ SNe. A recent bright example is SN 1994I in M51 (Filippenko et al. 1994).

It has been realized that what makes $\mathrm{SNe} \mathrm{Ib} / \mathrm{c}$ different must also extend to the nature of their progenitor stars (e.g., Wheeler \& Levreault 1985). Constraints on the progenitor can be obtained not only through observations of each event (see Leibundgut, these proceedings) and through theoretical modelling (see Nomoto, these proceedings), but also by examining the relationship of these $\mathrm{SNe}$ with their environment. Here we look at this environment on progressively smaller scales. Since only 32 bona fide members of this subclass exist, it must be kept in mind that small-number statistics are clearly a problem. Yet, even with this small sample, we are developing a clearer picture of the progenitor population for $\mathrm{SNe} \mathrm{Ib} / \mathrm{c}$. 


\section{Global Environment}

$\mathrm{SNe} \mathrm{Ib} / \mathrm{c}$ are found only in late-type galaxies (Porter \& Filippenko 1987; van den Bergh \& Tammann 1991). This implies that these SNe likely arise only from young massive stars, as do type II SNe. However, unlike SNe II, somehow during the lifetime of the progenitor star, the hydrogen envelope must have been entirely stripped away. Two hypotheses have been offered to accomplish this: (1) a Wolf-Rayet (W-R) star progenitor, or, (2) a progenitor in a massive interacting binary system. A previous discussion of W-R stars as $\mathrm{SNe} \mathrm{Ib} / \mathrm{c}$ progenitors can be found in Filippenko (1991). From the global standpoint, the rate of $\mathrm{SNe} \mathrm{Ib} / \mathrm{c}$ in these galaxies (van den Bergh \& Tammann 1991) is in conflict with hypothesis (1), since W-R stars, which evolve from stars with $M_{\mathrm{ZAMS}} \gtrsim 25-40 \mathrm{M}_{\odot}$ (Maeder \& Conti 1994), cannot provide enough progenitors.

\section{Local Interstellar Environment}

Anecdotally, it is known that $\mathrm{SNe} \mathrm{Ib} / \mathrm{c}$ tend to occur in or near $\mathrm{HII}$ regions (e.g., Porter \& Filippenko 1987). Kennicutt et al. (1989) showed that giant HII regions in late-type galaxies are the sites of the majority of massive star formation. Thus, if one finds $\mathrm{SNe}$ in or near $\mathrm{HII}$ regions, it is likely that their progenitors were massive stars. Van Dyk (1992) examined the association of $\mathrm{SNe}$ of all types, including $9 \mathrm{SNe} \mathrm{Ib/c}$, with $\mathrm{H} \mathrm{II} \mathrm{regions}$ in late-type galaxies. Recently, Van Dyk \& Hamuy (1993) extended this survey, considering $31 \mathrm{SNeII}$ and $15 \mathrm{SNe} \mathrm{Ib} / \mathrm{c}$. Both studies found little difference between the association of $\mathrm{SNe} \mathrm{Ib} / \mathrm{c}$ and of SNe II with $\mathrm{HII}$ regions. Here, we continue to explore this with a sample of $16 \mathrm{SNe} \mathrm{Ib} / \mathrm{c}$.

$\mathrm{HII}$ regions are mapped through narrow-band $\mathrm{H} \alpha$ imaging. Association of SNe with H II regions is established by comparing the projected angular separation $(\Delta)$ of each SN from the center of its nearest H II region with the maximum angular radial extent $\left(r_{\max }\right)$ of the $\mathrm{HII}$ region measured toward the SN position. Continuum-subtracted, flux-calibrated CCD data on nearly face-on galaxies have been acquired at KPNO, CTIO, Lowell Observatory, and Lick Observatory, as well as from other investigators. The $\mathrm{H}$ II region detection limit is $Z(2-3) 10^{37} \mathrm{erg} \mathrm{s}^{-1}$. We have measured $r_{\max }$ at a surface brightness level $\gtrsim 31^{-18} \mathrm{erg} \mathrm{cm}^{-2} \mathrm{~s}^{-1} \operatorname{arcsec}^{-2}$. As in Van Dyk \& Hamuy (1993), we use absolute positions or precise nuclear offsets when available. Astrometry for the CCD images with $\lesssim 1^{\prime \prime}$ accuracy is achieved using star positions on the images measured with the STScI GASP ${ }^{1}$ system.

\footnotetext{
${ }^{1}$ GASP is the Guide Star Astrometric Support Program available at the Space Telescope Science Institute (STScI).
} 

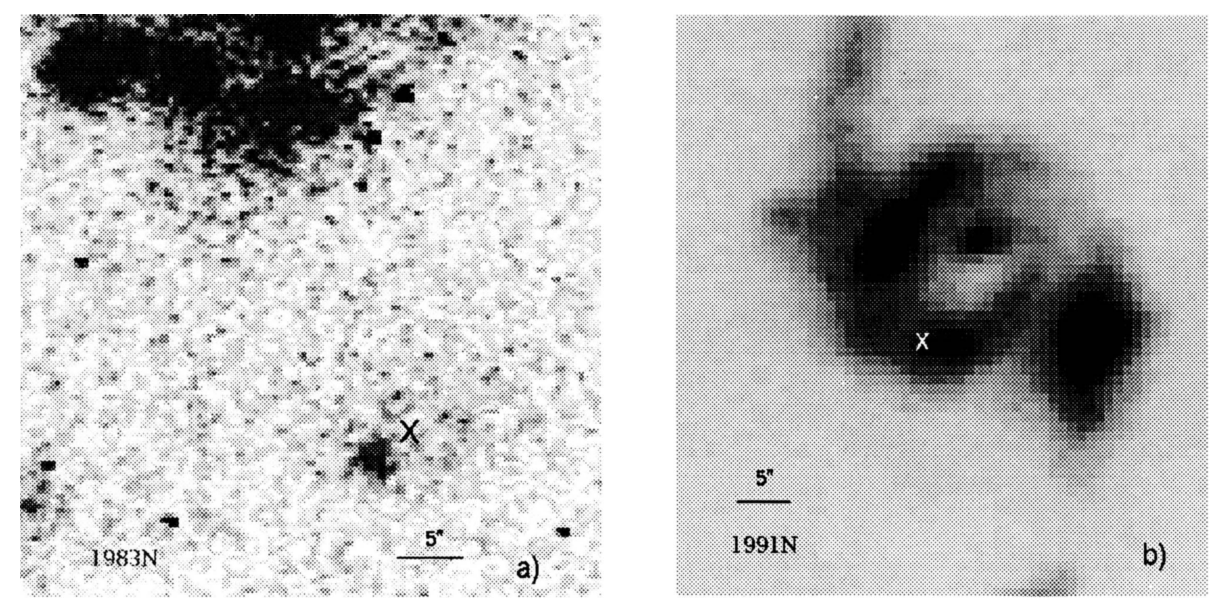

Figure 1. H $\alpha$ images of the environments of (a) the type Ib SN $1983 \mathrm{~N}$ in M83 and (b) the Type Ic SN 1991N in NGC 3310, showing the differing relationships of these two SNe with nearby H II regions. North is up, and East is to the left.

In Fig. 1 we show the local interstellar environments of the two $\mathrm{SNe} \mathrm{Ib} / \mathrm{c}$ $1983 \mathrm{~N}$ and $1991 \mathrm{~N}$.

For each $\mathrm{SN} \mathrm{Ib} / \mathrm{c}$ we determine the ratio $R \equiv \Delta / r_{\max }$. We then consider the proportion of $\mathrm{SNe} \mathrm{Ib} / \mathrm{c}$ with $R \leq 1$. Unfortunately, not all $\mathrm{SNe}$ in our sample can be treated equally. For lack of a better weighting scheme when calculating this proportion, those SNe having absolute positions or precise nuclear offsets are arbitrarily given 3 times the weight of those $\mathrm{SNe}$ before about 1980 with poorer nuclear offsets and 1.5 times the weight of those SNe after about 1980 with poorer offsets. (What are needed, of course, are accurate positions for all $\mathrm{SNe}$.) We find this proportion to be $0.57 \pm 0.12$ (statistical error) for $\mathrm{SNe} \mathrm{Ib} / \mathrm{c}$. Comparing this to $0.59 \pm 0.09$ of $32 \mathrm{SNe}$ II with $R \leq 1$, measured and weighted in the same manner, we find no significant difference. This suggests that the progenitors for both types may be very similar in ZAMS mass range, probably $8 \lesssim M\left(\mathrm{M}_{\odot}\right) \lesssim 25-40$. $\mathrm{W}$-R star progenitors are less likely, since a closer association of $\mathrm{SNe} \mathrm{Ib} / \mathrm{c}$ with HII regions might be expected for consistency with this hypothesis, although not all W-R stars in galaxies will necessarily be associated with bright starburst $\mathrm{H}$ II regions.

In addition to the lower-resolution ground-based data, we have also examined two $\mathrm{SNe} \mathrm{Ib} / \mathrm{c}$ environments using pre-repair Hubble Space Telescope (HST) archive data. These are broad-band, rather than narrow-band, images. In Fig. 2a we show a 200-s FOC UV image through the F220W filter for the site of SN $1991 \mathrm{~N}$. The site is midway between two probable large clusters of O-type stars. Photometry and improvement of the astrometry 

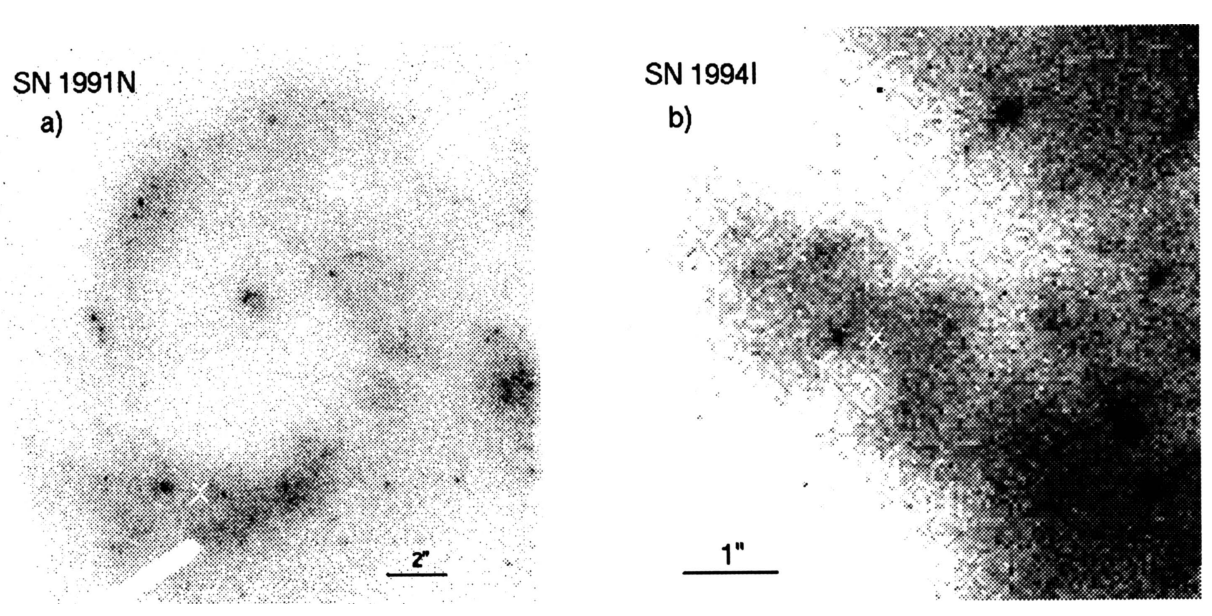

Figure 2. HST images of the environments of the type Ic supernovae (a) $1991 \mathrm{~N}$ in NGC 3310 and (b) 1994I in M51. North is up, and East is to the left.

is being done for this image.

In Fig. 2b we show a 1100-s PC image made through the F555W filter for the site of SN 1994I. We find a diffuse background of unresolved stars at the site. We estimate an upper limit $(3 \sigma)$ for the progenitor of $V \gtrsim 23.5 \mathrm{mag}$. Assuming a distance of $6.8-9.7 \mathrm{Mpc}$ and $A_{V} \simeq 1.5-2.0 \mathrm{mag}$ (Ho \& Filippenko 1995), the progenitor had $M_{V} \gtrsim-7.7$ to -8.4 mag. Unfortunately, this upper limit to the luminosity is not very restrictive; it is brighter than supergiants and many W-R stars (e.g., Torres-Dodgen \& Massey 1988).

Based on just these two HST images we can neither eliminate the W-R hypothesis nor support the competing massive binary hypothesis. Additional archive images, especially the more valuable post-repair ones, will soon become available. Inevitably, HST imaging will be the best way to detail the interstellar and stellar environments for $\mathrm{SNe}$ and begin to place rigorous mass constraints on the progenitors. In the cases of SNe $1983 \mathrm{~N}$ and $1994 \mathrm{I}$ it should also eventually be possible to locate, through HST imaging, any possible surviving binary companion to the progenitor.

\section{Circumstellar Environment}

$\mathrm{SNe} \mathrm{Ib} / \mathrm{c}$, like SNe II, are radio emitters (Weiler et al. 1986). This was first discovered for SN 1983N by Sramek et al. (1984). The other cases include $\mathrm{SNe} 1984 \mathrm{~L}, 1990 \mathrm{~B}$, and 1994I. Most radio supernovae (RSNe) share the common properties of non-thermal emission with high brightness temperature, light curve "turn-on" at shorter wavelengths first and longer wavelengths later, a rapid increase in flux density with time at each wavelength, with a power-law decline after maximum, and a decreasing spectral index 

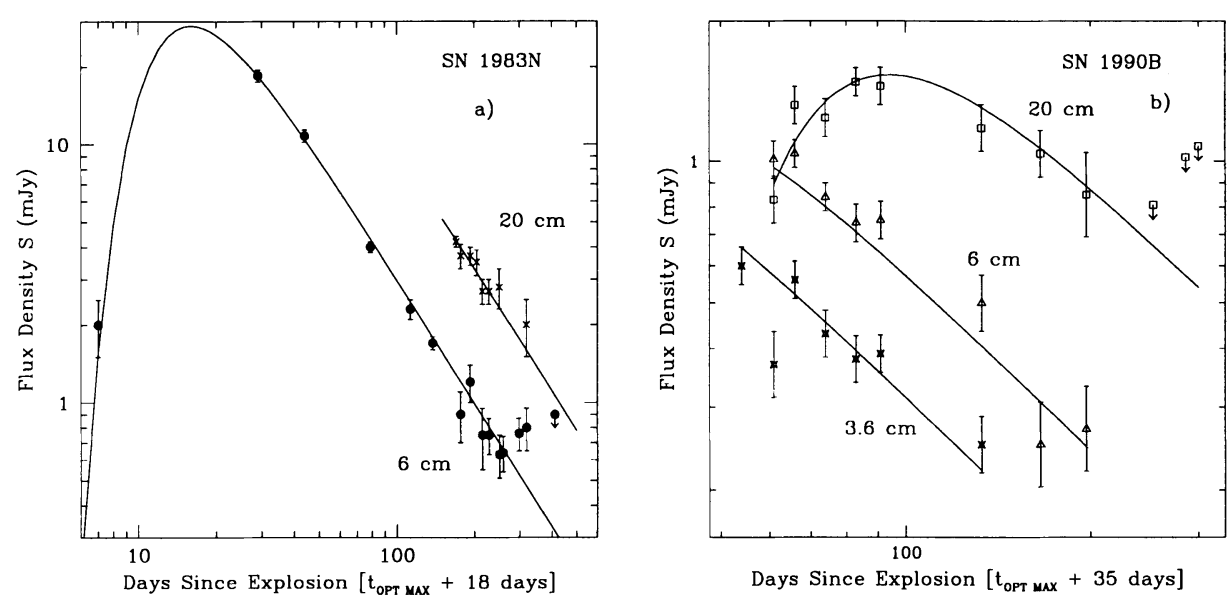

Figure 3. Radio light curves for (a) the type Ib SN 1983N (Weiler et al. 1986) and (b) the type Ic SN 1990B in NGC 4568 (Van Dyk et al. 1993).

between two wavelengths, with the spectral index $\alpha$ inevitably approaching an optically thin, non-thermal, constant negative value (Weiler et al. 1986). $\mathrm{RSNe} \mathrm{Ib} / \mathrm{c}$ show a steep spectral index, rapid "turn-on" at $6 \mathrm{~cm}$ before optical maximum, rapid decline after maximum, and homogeneity in spectral luminosity at $6 \mathrm{~cm}\left(\sim 10^{27} \mathrm{erg} \mathrm{s}^{-1} \mathrm{~Hz}^{-1}\right)$. This behavior is in contrast to the slower, more gradual, more heterogeneous behavior for RSNe II.

In Fig. 3 we show radio light curves for the two well-studied $\mathrm{RSNe} \mathrm{Ib} / \mathrm{c}$ $1983 \mathrm{~N}$ and 1990B.

The radio emission from $\mathrm{SNe}$ has been successfully interpreted using the Chevalier (1984) "mini-shell" model, where non-thermal synchrotron emission is produced via interaction of the SN shock with the high-density circumstellar matter produced by a red supergiant progenitor star through mass-loss in the form of a stellar wind prior to explosion. The synchrotron emission is assumed to be free-free absorbed by the fully ionized wind matter. Both the synchrotron luminosity and the absorption depend on the circumstellar density, which is proportional to the ratio of the mass-loss rate $\dot{M}$ to the wind speed $w$ (Weiler et al. 1986) for a spherically symmetric wind. For $\mathrm{RSNe} \mathrm{Ib} / \mathrm{c}, \dot{M} / w \approx 10^{-6} \mathrm{M}_{\odot} \mathrm{yr}^{-1} /\left(\mathrm{km} \mathrm{s}^{-1}\right)$. For a W-R star with a fast $\left(w \sim 10^{3} \mathrm{~km} \mathrm{~s}^{-1}\right)$, low-density wind, the required $\dot{M}\left(\sim 10^{-3} \mathrm{M}_{\odot} \mathrm{yr}^{-1}\right)$ is much larger than typically observed for these stars (e.g., Conti 1988), and would result in a low-density bubble around the star prior to explosion, rather than a high-density shell necessary to produce the rapidly evolving, luminous radio emission. We can then reasonably exclude the $\mathrm{W}-\mathrm{R}$ model. The homogeneity in radio properties for $\mathrm{SNe} \mathrm{Ib} / \mathrm{c}$ implies a homogeneity 
in circumstellar environments, which must be different from SNe II circumstellar environments. Interaction in a massive binary system could provide this difference.

Acknowledgements. This work was partially based on observations made with the NASA/ESA HST, obtained from the data archive at STScI, which is operated by the Association of Universities for Research in Astronomy, Inc., under NASA contract NAS 5-26555. Financial support was provided by NASA through grant number AR-4302.01-92A from STScI.

\section{References}

Branch, D. 1986, ApJ 300, L51

Chevalier, R.A. 1984, ApJ 285, L63

Conti, P.S. 1988, in O Stars \& Wolf-Rayet Stars, P.S. Conti \& A.B. Underhill (Eds.), p. 81

Elias, J.H., Matthews, K., Neugebauer, G. \& Persson, S.E. 1985, ApJ 296, 379

Filippenko, A.V. 1986, in Highlights of A stronomy, J.-P. Swings (Ed.), Reidel (Dordrecht), p. 589

Filippenko, A.V. 1991, in Wolf-Rayet Stars and Interrelations with Other Massive Stars in Galaxies, K.A. van der Hucht \& B. Hidayat (Eds.), Kluwer (Dordrecht), p. 529

Filippenko, A.V., Matheson, T. \& Barth, A.J. 1994, IAU Circ. 5964

Harkness, R.P. et al. 1987, ApJ 317, 355

Ho, L.C. \& Filippenko, A.V. 1995, ApJ (in press)

Kennicutt, R.C., Jr., Edgar, B.K. \& Hodge, P.W. 1989, ApJ 337, 761

Maeder, A. \& Conti, P.S. 1994, ARA\&A 32, 227

Porter, A.C. \& Filippenko, A.V. 1986, AJ 93, 1372

Sramek, R.A., Panagia, N. \& Weiler, K.W. 1984, ApJ 285, L59

Torres-Dodgen, A.V. \& Massey, P. 1988, AJ 96, 1076

Uomoto, A. \& Kirshner, R.P. 1985, A\&A 149, L7

van den Bergh, S. \& Tammann, G.A. 1991, ARA\&A 29, 363

Van Dyk, S.D. 1992, AJ 103, 1788

Van Dyk, S.D., \& Hamuy, M. 1993, in Massive Stars: Their Lives in the Interstellar Medium, J.P. Cassinelli \& E.B. Churchwell (Eds.), ASP Conf. Ser. Vol. 35, p. 440

Van Dyk, S.D., Sramek, R.A., Weiler, K.W. \& Panagia, N. 1993, ApJ 409, 162

Weiler, K.W., Sramek, R. A., Panagia, N., van der Hulst, J.M. \& Salvati, M. 1986, ApJ 301,790

Wheeler, J.C. \& Harkness, R. P. 1990, Rept. Progr. Phys. 53, 1467

Wheeler, J.C. \& Levreault, R. 1985, ApJ 294, L17 